\title{
Asociación entre nomofobia e indicadores de inteligencia en estudiantes de educación superior
}

\author{
Quitzia Torres-Salazar',2, Alberto Ramírez-Gurrola', Michelle Castañón-Alvarado', Acis Aroña-Campos', Arely \\ Betancourt-Araujo', María Fernanda Carrillo-Cisneros', Christian López-Molina', Juan Gerardo Medina-Rosales', \\ Bulmaro Carrillo de la Cruz' \\ I Escuela de Ciencias Médicas, Universidad Autónoma España de Durango, Durango, México \\ 2 Núcleo de Nutrición y Enfermedades Metabólicas, Universidad Autónoma España de Durango, Durango, México
}

\section{RESUMEN}

Introducción: el uso indiscriminado de los teléfonos móviles puede estar asociado a eventos de igual forma negativos como insomnio, depresión, estrés o tendinitis crónica. La identificación de factores emocionales de riesgo para el desarrollo de nomofobia es importante para poder priorizar estrategias de intervención preventiva en adolescentes, quienes dicho sea de paso se unen cada vez más al grupo de personas adictas al celular o como se define en este estudio, con nomofobia. Objetivo: determinar si existe relación entre la presencia de nomofobia e indicadores de inteligencia entre ellos: a) Inteligencia cognitiva medida a través del IQ; b) Resiliencia; y c) Autoestima. Método: diseño observacional, analítico, transversal, prospectivo, de Casos y Controles pareados por edad y sexo. La muestra se integró por 70 casos y 70 controles, con una edad promedio de 22 ( $D E$ = 1.5 años); $60 \%$ hombres y $40 \%$ mujeres. Resultados: se encontró que en el grupo de personas con IQ promedio o por debajo del promedio es 27 veces más probable el desarrollo de nomofobia, comparado con el grupo de no nomofóbicos. Al realizar este ejercicio con la baja autoestima, se identificó un OR de 58, y con resiliencia promedio o baja, el OR es de 2.2 (ambos con IC 95\% estadísticamente significativos). Conclusión: existe asociación entre la presencia de nomofobia e indicadores de inteligencia cognitiva y emocional.

Palabras clave: nomofobia, tecnopatías, enfermedades mentales.

\begin{abstract}
Introduction: the indiscriminate use of mobile phones may be associated with equally negative events such as insomnia, depression, stress or chronic tendonitis. The identification of emotional risk factors for the development of nomophobia is important in order to prioritize preventive intervention strategies in adolescents, who by the way are increasingly joining the group of people addicted to cell phones or as defined in this study, with nomophobia. Objective: this study has the objective of determinate if there are any correlationship between the presence of nomophobia and intelligence indicators including a) Cognitive intelligence measured by $I Q, b)$ Resilience \& c) Self-esteem. Methods: this study have an observational, analytic, transversal, prospective design of case-control study paired by age and sex. The sample was composed of 70 cases and 70 controls, with an average age of 22 ( $S D=1.5$ years), being $60 \%$ males and $40 \%$ females. Results: in the group of people with average or below average IQ, the development of nomophobia was 27 times more probable compared to the non-nomophobic group. When performing this exercise with low self-esteem was identified an OR of 58 and with average or low resilience the OR is 2.2 (both with 95\% statistically significant IC). Conclusion: there is an association between the presence of nomophobia and indicators of cognitive and emotional intelligence.
\end{abstract}

Keywords: nomophobia, technopathology, mental disorders.

\footnotetext{
Autor de correspondencia:

Quitzia Torres-Salazar. Playa Roqueta núm. 231, Fracc: Las Playas Durango, Durango, México. Teléfono (01618) 8230924. Correo electrónico: quitzia.torres@gmail.com.

Recibido: 25 de junio de 2018

Aprobado: 28 de agosto de 2018

doi: 10.28931/riiad.2018.2.03
} 


\section{INTRODUCCIÓN}

La nomofobia (No mobile Phone Phobia) ha sido considerada como un tema controversial, ya sea que se le defina como una dependencia del teléfono móvil (Dixit et al., 2010) o la adicción a los teléfonos móviles (Forgays, Hyman, \& Schreiber, 2014). La nomofobia es una tecnopatía del mundo moderno (Wang et al., 2014) definida como un sentimiento de incomodidad, ansiedad, nerviosismo o angustia que resulta de no estar en contacto con el teléfono móvil, incluso llegando a detonar ideación suicida. Arpaci estudia en específico la relación que existe entre la nomofobia y el apego emocional, y concluye que las personas que son emocionalmente más dependientes y desean más cercanía y atención, tienden a mostrar niveles más altos de miedo o incomodidad al no estar cerca de sus teléfonos móviles (Arpaci, Baloğlu, Özteke Kozan, \& Kesici, 2017); de igual forma, destaca diferencias de esta entidad emocional relacionada al género, las mujeres presentan mayor apego evitativo vinculado con nomofobia.

En las últimas décadas, los teléfonos móviles se han convertido en una herramienta indispensable con capacidades que superan con creces su propósito original. Por lo tanto, los teléfonos inteligentes no sólo permiten a los usuarios llamar o enviar mensajes de texto a otras personas, sino que también proporcionan acceso a Internet y a todos los servicios disponibles en él (Canovas et al., 2014). Según varias encuestas de INEGI, 59.5\% de la población mayor de seis años en México tiene acceso a un teléfono inteligente (ENDUTIH, 2016). Asimismo, se describe que $68.5 \%$ de los cibernautas mexicanos tiene menos de 35 años y que $47.0 \%$ de los hogares del país tiene conexión a Internet.

Según la ENDUTIH, las Tecnologías de la Información y las Comunicaciones (TIC) constituyen herramientas privilegiadas para el desarrollo de los individuos y de las sociedades, al facilitar el manejo de información: crearla, compartirla, modificarla, enriquecerla y transformarla en conocimiento. El uso de las TIC se encuentra cada vez más difundido tanto por la aparición de nuevos dispositivos como por la reducción de los costos asociados. De la población que cuenta con estudios de nivel superior (licenciatura o posgrado), nueve de cada 10 han incorporado el uso de Internet en sus actividades habituales; cuatro de cada cinco de los que cuentan con estudios de nivel medio superior (preparatoria o equivalente) también lo hacen, y con nivel básico (primaria o secundaria) resulta poco menos de la mitad $(48.7 \%)$.

La inteligencia cognitiva entendida como el conjunto de las habilidades cognitivas o intelectuales necesarias para obtener conocimientos y utilizar esos conocimientos de forma correcta, con el fin de resolver problemas que tengan un objetivo y una meta bien descritos (Resing \& Drent, 2001), debería ser parte de la anamnesis integral, a fin de entender la vulnerabilidad de un individuo para desarrollar algunos desórdenes del tipo de la nomofobia. Aunque se ha identificado relación de la nomofobia con la carencia de inteligencia emocional, no se cuenta con datos suficientes que la asocien con inteligencia cognitiva y para fines diagnósticos; a pesar de que se ha propuesto su incorporación al DSM-V, la nomofobia no se ha reconocido de manera formal como una patología (Bragazzi \& Del Puente, 2014). No obstante, la clave para saber si realmente existe un desorden es tener en cuenta la repercusión en la vida diaria. Si la dependencia al smartphone está afectando la vida social y laboral, el rendimiento escolar, las relaciones de pareja o cualquier otra área de la vida, puede tratarse de un problema de nomofobia.

Desde el contexto de la salud y las adicciones, es de primordial importancia desarrollar el constructo de vulnerabilidad para poder hacer frente a su impacto en los problemas de salud y en los comportamientos de consumo químico y no químico. Teóricamente, interviene en la construcción de la vulnerabilidad psicosocial una serie de variables: riesgo, afrontamiento, resiliencia, estrés, apego e inteligencia emocional, que modulan su configuración y consiguen que al final la vulnerabilidad se transforme en un factor de riesgo o de protección en función de cómo actúen dichas variables en la fase final (García del Castillo, 2015). En este contexto, la inteligencia cognitiva, así como la resiliencia y una elevada autoestima fungirían como factores protectores ante el desarrollo de conductas adictivas.

Los nomofóbicos, sean de la edad que sean, suelen tener la necesidad de obtener respuestas inmediatas, de modo que cuando no sucede así, repercute negativamente en su autoestima y seguridad en sí mismos (Carbonell, 2015).

Una de las principales preocupaciones se centra en el aislamiento social al que puede conducir el abuso del móvil o Internet en general. Esto puede suceder especialmente en muchos adolescentes que restringen su vida social al uso de este tipo de dispositivos, lo que resulta en una pérdida de habilidades sociales. Además, la dependencia suele interferir en sus horarios, retrasa la hora de irse a dormir y afecta a su rendimiento escolar.

Este estudio tuvo el objetivo de determinar si existe relación entre la presencia de nomofobia e indicadores de inteligencia, entre los cuales se incluyen: a) Inteligencia cognitiva medida a través del IQ; b) Resiliencia; y c) Autoestima. 


\section{MÉTODO}

\section{Aspectos éticos}

Todos los datos obtenidos en esta investigación cumplen con la obtención de consentimiento informado de los participantes.

\section{Diseño del estudio}

Tuvo un diseño observacional, analítico, transversal, prospectivo, de Casos y Controles pareados por edad y sexo.

\section{Universo de estudio}

Estudio estuvo constituido por alumnos de nivel profesional de una institución de nivel superior del estado de Durango.

\section{Participantes}

Se seleccionó a 70 estudiantes para cada grupo, con un método aleatorizado y estratificado por semestres del Instituto Tecnológico de Durango.

\section{Variables de estudio e instrumentos de medición}

1. Nomofobia (Ramos, López, \& Quiles, 2017). Medida con la escala de Yildrim, entendida como una adicción comportamental que implica un alto temor a quedarse sin teléfono móvil; para fines de este estudio se utilizó de manera dicotómica diagnosticando como positivo la obtención de una puntuación de 67 o más.

2. Autoestima (Góngora \& Casullo, 2009). Medida con la escala de Rosemberg, entendida como los sentimientos de valía personal y de respeto a sí mismo; se aplicó un cuestionario para explorar la autoestima personal, considerando autoestima baja una puntuación de menos de 25 puntos, autoestima media 26 a 29 puntos y autoestima alta arriba de 30 puntos.

3. Resiliencia (Teruel \& Robles, 2015). Medida con la escala de Sánchez adaptada al ser humano, resiliencia es la capacidad de prevalecer, crecer, ser fuerte y hasta triunfar a pesar de las adversidades (Salgado, 2005). Caracteriza a aquellas personas que, a pesar de vivir situaciones de alto riesgo o sufrir situaciones adversas, se desarrollan psicológicamente sanas y exitosas, e incluso salen fortalecidas de estas situaciones (Páez et al., 2011). Para fines de este estudio se clasificaron en cinco niveles: 1) Muy bajo (puntuación de 0 a 15); 2) Bajo (19-23); 3) Promedio (24-33); 4) Alto (34-42); y 5) Muy alto (43 o más).
4. Coeficiente Intelectual (Amador, 2013). Medida con la escala de Wechsler, entendida como la capacidad de una persona para actuar con una finalidad, para pensar racionalmente y desenvolverse con eficacia dentro de su ambiente; clasificada en IQ por debajo del promedio, IQ promedio, IQ superior al promedio e IQ superior.

\section{Muestra}

Se determinó una muestra de 70 personas para cada grupo, considerando un valor alfa de 0.05 y un poder del $80 \%$.

\section{Análisis estadístico}

Para el análisis descriptivo se determinaron medidas de tendencia central y dispersión; para la comparación de medias se utilizó la $t$ de Student (en caso de comportamiento paramétrico) y U de Mann Whitney (en caso de comportamiento no paramétrico); la comparación entre porcentajes se realizó con la prueba de Chi cuadrada. Se categorizaron los puntajes obtenidos para realizar un análisis de contingencia con determinación de OR.

\section{RESULTADOS}

Se aplicaron 200 encuestas a estudiantes del Instituto Tecnológico de Durango y se identificaron las que presentaron un diagnóstico de nomofobia, a partir de las cuales se obtuvieron de manera aleatoria 70 casos; los 70 controles se integraron con sujetos identificados sin nomofobia.

Los grupos quedaron conformados por adultos jóvenes de entre 18 y 30 años. Se eliminaron de la muestra aquellas encuestas incompletas o invalidadas por el propio instrumento. La edad promedio de la muestra fue de 22 ( $D E=1.5$ años); 60\% fueron hombres y $40 \%$, mujeres, ambas características sin diferencias entre grupos.

Se indagó por el número de selfies que se acostumbraba tomar la persona diariamente así como la publicación de estas en redes sociales con una considerable diferencia entre las personas con nomofobia (29 selfies y 9 publicaciones por semana) vs personas sin nomofobia (cuatro selfies y una publicación; Tabla 1).

De igual forma, se interrogó acerca del número de likes esperados por publicación realizada en redes sociales y se encontraron diferencias estadísticamente significativas entre grupos (100 vs 30).

Se realizó una estratificación de los resultados, a fin de identificar los elementos de riesgo: estrato I (aquellos que presentaron IQ por encima del promedio o superior) vs estrato II (aquellos con IQ promedio o por debajo del 
Tabla 1

Características generales de la población en estudio. Diferencias entre grupos

\begin{tabular}{|c|c|c|c|c|c|}
\hline \multirow[b]{2}{*}{ Sexo } & \multicolumn{2}{|c|}{$\begin{array}{c}\text { Con nomofobia } \\
n=70\end{array}$} & \multicolumn{2}{|c|}{$\begin{array}{c}\text { Sin nomofobia } \\
n=70\end{array}$} & \multirow{2}{*}{$\begin{array}{c}p \\
0.806\end{array}$} \\
\hline & $\mathrm{H}(61.4 \%)$ & M (38.6\%) & $H(60 \%)$ & M (40\%) & \\
\hline Edad $^{*}$ & 22.18 & $(1.5)$ & 22.27 & $(1.5)$ & 0.743 \\
\hline Selfies tomadas por semana** & 29 & $(18-44)$ & 4.5 & $(2-6)$ & 0.000 \\
\hline Selfies subidas a FB ${ }^{1}$ por semana ** & 9 & $(6-14)$ & 1 & $D E(0.05-1)$ & 0.000 \\
\hline No. De likes esperados por publicación** & 100 & $(80-150)$ & 30 & $(20-40)$ & 0.000 \\
\hline
\end{tabular}

NOTA: * Media y SD. ** Mediana y Cuartiles 25 y 75. Las diferencias se estimaron con la prueba T de Student. ${ }^{1}$ Facebook. La diferencia entre medianas se estimó con prueba $U$ de Mann Withney

Fuente: encuesta directa

Tabla 2

Clasificación en la puntuación de coeficiente intelectual y nivel de autoestima en estudiantes universitarios con nomofobia y sin nomofobia

\begin{tabular}{|c|c|c|c|c|c|c|c|}
\hline Estrato I & \multicolumn{2}{|c|}{$\begin{array}{l}\text { Con nomofobia } \\
n=70\end{array}$} & \multicolumn{2}{|c|}{$\begin{array}{c}\text { Sin nomofobia } \\
n=70\end{array}$} & $p$ & OR & IC 95\% \\
\hline IQ Superior* & - & - & 1 & $(1.4)$ & & & \\
\hline IQ Por encima del promedio & 1 & $(1.4)$ & 19 & $(27)$ & 0.000 & 27.6 & $3.58-212$ \\
\hline \multicolumn{8}{|l|}{ Estrato II } \\
\hline IQ Promedio & 36 & (51) & 46 & (65) & & & \\
\hline IQ Por debajo del promedio & 33 & $(47)$ & 4 & $(5.7)$ & & & \\
\hline \multicolumn{8}{|l|}{ Estrato I } \\
\hline Autoestima alta & 4 & $(5.7)$ & 48 & (68) & & & \\
\hline Autoestima promedio & 34 & $(48.5)$ & 21 & (30) & 0.000 & 58.1 & $7.6-442$ \\
\hline \multicolumn{8}{|l|}{ Estrato II } \\
\hline Autoestima baja & 32 & $(45.7)$ & 1 & $(1.4)$ & & & \\
\hline
\end{tabular}

Nota: * Se introdujo el Estrato II en la tabla de contingencia como representante del grupo de expuestos

Fuente: encuesta directa. Los datos son presentados como $n(\%)$

Tabla 3

Características de resiliencia en la población de estudio. Diferencias entre grupo

\begin{tabular}{|c|c|c|c|c|c|}
\hline & $\begin{array}{c}\text { Con nomofobia } \\
n=70\end{array}$ & $\begin{array}{c}\text { Sin nomofobia } \\
n=70\end{array}$ & $p$ & OR & IC 95\% \\
\hline \multicolumn{6}{|l|}{ Estrato I } \\
\hline Muy alta & $4(5.7)$ & $1(1.4)$ & & & \\
\hline Alta & 17 (24.3) & $33(47.1)$ & & & \\
\hline \multicolumn{6}{|l|}{ Estrato II } \\
\hline Promedio & $28(40)$ & $26(37.1)$ & 0.012 & 2.2 & $1.1-4.4$ \\
\hline Baja & $21(30)$ & $10(14.3)$ & & & \\
\hline
\end{tabular}

Nota: * la diferencia entre porcentajes se calculó con chi cuadrada

Fuente: encuesta directa. Los datos representan la $n(\%)$ 
promedio (Tabla 2); se encontró un OR de 27.6 veces más probabilidades de presentar nomofobia en el estrato I.

Se realizó el mismo ejercicio con la autoestima: se identificó un OR de 58 veces más probabilidades de desarrollar nomofobia en el estrato I, ambos con IC 95\% significativo.

En la Tabla 3 podemos observar cómo tener características de resiliencia baja o promedio incrementa 2.2 veces la probabilidad de desarrollar nomofobia, comparado con el grupo donde la resiliencia es alta o muy alta.

\section{DISCUSIÓN Y CONCLUSIONES}

Numerosos estudios han explorado cómo los rasgos de personalidad contribuyen al inicio y mantenimiento de los trastornos de adicción en adultos jóvenes, con altas puntuaciones de impulsividad y bajo autocontrol; estos factores son clave en el desarrollo de la adicción (Lee et al., 2012). En concordancia, en este estudio se destaca que un buen nivel de autoestima y resiliencia, así como un coeficiente intelectual elevado son factores protectores ante el riesgo de desarrollar cualquier tipo de adicción, y en lo relacionado con la nomofobia, los resultados lo exponen en términos de un OR estadísticamente significativo de 27,58 y 2.2 respectivamente.

La adicción a Internet se define como la incapacidad de controlar su uso y es una condición que da como resultado el deterioro grave de varias funciones de la vida (Becoña \& Oblitas, 2002). En este estudio se evidencia al número de selfies tomado como un indicador con respecto a la presencia de nomofobia; en concordancia, otros estudios (Janarthanan et al., en 2018) resaltan la relación que existe entre el autorretrato o la toma de selfies con el trastorno narcisista al mismo tiempo (McCain et al., 2016). Se ha detectado, en este contexto, que el atractivo social era la principal motivación para publicar selfies. Charoensukmongkol informó que la búsqueda de atención, la soledad y el comportamiento egocéntrico tuvo una relación significativa con el gusto por los autorretratos (Charoensukmongkol, 2016). Se expone además, la estrecha relación que existe entre la nomofobia y la toma de selfies, mostrando una razón 6.5: 1 entre nomofóbicos y no nomofóbicos.

Los hallazgos coinciden con la hipótesis planteada por Olivencia-Carrión (Olivencia-Carrión, Ferri-García, Rueda, Jiménez-Torres, \& López-Torecillas, 2018), quien destaca que hay variables de personalidad (temperamento y carácter) que protegen contra la aparición de la nomofobia. Las variables de temperamento se reflejarían en puntajes bajos en la búsqueda de novedad, evitación de daño, dependencia de recompensa y persistencia, mientras que las variables de carácter estarían representadas por puntuaciones altas en autodirección, coopera- ción y autotrascendencia, y viceversa para el riesgo del desarrollo de la nomofobia.

Es importante destacar que de los tres factores de inteligencia estudiados, dos de ellos (autoestima y resiliencia) son potencialmente modificables, posibilitando el proceso de la aparición de nomofobia o incluso prevenirlo de manera efectiva con actividades encaminadas mejorar o fortalecer tanto el perfil de resiliencia como de autoestima. Se considera factible proponer que la nomofobia sea considerada como una alteración seria, que de identificarse y atenderse de manera oportuna brinda la posibilidad, especialmente al docente, de incrementar el rendimiento escolar y al personal de salud de prevenir adicciones alternas $u$ otras enfermedades mentales.

De los resultados obtenidos, se puede concluir que el número de selfies tomadas al día puede ser una referencia confiable en la búsqueda de nomofobia. Asimismo, se puede asegurar que los indicadores de inteligencia emocional y cognitiva, como son resiliencia, autoestima y coeficiente intelectual, están asociados a la presencia de nomofobia, por lo que su identificación temprana puede ser de utilidad para la selección de población para intervenciones focales con impacto en la salud mental y desempeño académico.

Una limitante de este estudio fue la falta de inclusión de la nomofobia como un trastorno mental reconocido en el DSM-V, lo cual limita su diagnóstico a los instrumentos validados por organismos particulares, por lo menos hasta el momento de la definición de las variables en el estudio. Se propone para nuevos estudios tomar en cuenta nuevos instrumentos, por ejemplo la propuesta de Wang (Wang et al., 2014), que con su equipo valida un nuevo inventario de adicción al smartphone con un buen nivel de confiabilidad.

\section{CONFLICTOS DE INTERÉS}

Los autores declaran que esta investigación fue financiada con recursos propios y que no existe conflicto de interés ni propio ni de terceros.

\section{REFERENCIAS}

Amador, J. (2013). Escala de inteligencia de Wechsler para adultos, cuarta edición (WAIS-IV). Universidad de Barcelona.

Arpaci, I., Baloğlu, M., Özteke Kozan, H. I., \& Kesici, Ș. (2017). Individual Differences in the Relationship Between Attachment and Nomophobia Among College Students: The Mediating Role of Mindfulness. Journal of Medical Internet Research, 19(12), e404. doi:10.2196/jmir.8847

Becoña E., \& Oblitas L., (2002) Adicciones y Salud. Revista Psicología Científica, 4(2). Recuperado de http://www.psicologiacientifica.com/adicciones-y-salud 
Bragazzi, N., \& Del Puente, G. (2014). A proposal for including nomophobia in the new DSM-V. Psychology Research and Behavior Management, 7. 155-160. doi:10.2147/prbm.s41386

Canovas, G., García-de-Pablo, A., Oliaga-San-Atilano, A., \& AboyFerrer, I., (2014) Menores de Edad y Conectividad Móvil en España: Tablets y Smartphones. España: Protégeles.

Carbonell, J., (2015) Nomofobia: Adicción al Smartphone. Diario de Mallorca: Salud mental y vida sana, Recuperado de https://www. diariodemallorca.es/blogs/salud-mental-y-vida-sana/nomofobiaadiccion-al-smartphone.html

Charoensukmongkol, P. (2016). Exploring personal characteristics associated with selfie-liking. Cyberpsychology: Journal of Psychosocial Research on Cyberspace, 10(2), 7. doi:10.5817/ cp2016-2-7

Dixit, S., Shukla, H., Bhagwat, A., Bindal, A., Goyal, A., Zaidi, A., \& Shrivastava, A. (2010). A study to evaluate mobile phone dependence among students of a medical college and associated hospital of central India. Indian Journal of Community Medicine, 35(2), 339-341. doi:10.4103/0970-0218.66878

Forgays, D. K., Hyman, I., \& Schreiber, J. (2014). Texting everywhere for everything: Gender and age differences in cell phone etiquette and use. Computers in Human Behavior, 31, 314-321. doi:10.1016/j.chb.2013.10.053

García del Castillo, J. (2015) Concepto de vulnerabilidad psicosocial en el ámbito de la salud y las adicciones, Health and addictions, 15(1), 5-14

Góngora, V., \& Casullo, M. (2009) Validación de la escala de autoestima de Rosenberg en población general y en población clínica de la Ciudad de Buenos Aires. Revista Iberoamericana de Diagnóstico y Evaluación, 1(27) 179-194.

Instituto Nacional de Estadística y Geografía (2016) Encuesta Nacional sobre Disponibilidad y Uso de Tecnologías de la Información en los Hogares (ENDUTIH )2016. México: Autor.

Janarthanan, B., \& Marck, D., (2018) An Exploratory Study of "Selfitis" and the Development of the Selfitis Behavior Scale. International Journal of Mental Health and Addiction, 16(3), 722-736.
Lee, H. W., Choi, J.-S., Shin, Y.-C., Lee, J.-Y., Jung, H. Y., \& Kwon, J. S. (2012). Impulsivity in Internet Addiction: A Comparison with Pathological Gambling. Cyberpsychology, Behavior, and Social Networking, 15(7), 373-377. doi:10.1089/cyber.2012.0063

McCain, J. L., Borg, Z. G., Rothenberg, A. H., Churillo, K. M., Weiler, P., \& Campbell, W. K. (2016). Personality and selfies: Narcissism and the Dark Triad. Computers in Human Behavior, 64, 126-133. doi:10.1016/j.chb.2016.06.050

Olivencia-Carrión, M. A., Ferri-García, R., Rueda, M. del M., Jiménez-Torres, M. G., \& López-Torrecillas, F. (2018). Temperament and characteristics related to nomophobia. Psychiatry Research, 266, 5-10. doi:10.1016/j.psychres.2018.04.056

Páez, D., Vázquez, C., Bosco, S., Gasparre, A., Iraurgi, I., \& Sezibera, V. (2011). Crecimiento post estrés y post trauma: Posibles aspectos positivos y beneficiosos de la respuesta a los hechos traumáticos. En D. Páez, C. Martín-Beristain, J. L. González, N. Basabe \& J. De Rivera (Eds.), Superando la violencia colectiva y construyendo cultura de paz (pp. 311-339). Madrid: Fundamentos

Ramos I., López C., \& Quiles M., (2017). Adaptación y validación de la escala de nomofobia de Yildirim y Correia en estudiantes españoles de la educación secundaria obligatoria. Health and Addictions, 17(2), 201-213.

Resing, W. C. M., \& Drenth, P. J. D. (2001), Intelligentie: Weten en meten. Amsterdam: Nieuwezijds.

Salgado, A. (2005). Métodos e instrumentos para medir la resiliencia: Una alternativa peruana. Revista de Psicología, 11, 41-48.

Teruel D., \& Robles M. (2015) 14-item Resilience Scale (RS-14): Psychometric Properties of the Spanish Version. Revista Iberoamericana de Diagnóstico y Evaluación, 2(40) 103-113.

Wang, P. W., Liu, T. L., Ko, C. H., Lin, H. C., Huang, M. F., Yeh, Y. C., \& Yen, C. F. (2014). Association between Problematic Cellular Phone Use and Suicide: The Moderating Effect of Family Function and Depression. Comprehensive Psychiatry, 55(2), 342-348. doi:10.1016/j.comppsych.2013.09.006 